\title{
GMR
}

\section{Comparative sequence and expression analysis of tapetum specific male sterility related genes in Medicago truncatula}

\author{
L.H. Shao, X.W. Zheng, D.X. Yi and C. Li \\ Institute of Animal Sciences, the Chinese Academy of Agricultural Sciences, \\ Beijing, China \\ Corresponding author: C. Li \\ E-mail: licong0520@sina.com
}

Genet. Mol. Res. 15 (2): gmr.15028323

Received December 21, 2015

Accepted March 11, 2016

Published July 15, 2016

DOI http://dx.doi.org/10.4238/gmr.15028323

\begin{abstract}
Heterosis, or enhancement through outbreeding, is one of the most promising approaches for increasing crop yield. Male sterility (MS), which promotes heterosis, has been widely applied in hybrid crop production. Medicago truncatula is a model legume species and is closely related to M. sativa, an important legume forage plant. Although the molecular mechanisms of MS in M. truncatula and M. sativa remain unclear, several studies of MS have been conducted in Arabidopsis thaliana. Previous research has shown that MS is associated with the destruction of tapetal cell layers. Disruption of tapetum developmental processes may result in pollen abortion. In an effort to identify genes useful for breeding in $M$. sativa, we identified MS related genes in $M$. truncatula using BLAST and homology to $A$. thaliana genes. In this study, we identified 63 tapetum specific male sterility (TSMS) related genes. The length of TSMS genes varied from 225 to $3747 \mathrm{bp}$. We identified 15 conserved domains and 8 cis-elements associated with TSMS related genes. Analysis of the phylogenetic relationships among these genes allowed them to be classified into three groups, MtTsms A, MtTsms B, and MtTsms C. Expression analyses revealed that these
\end{abstract}


genes may be involved in developmental processes and response to abiotic stress.

Key words: Medicago truncatula; Male sterility; Genetic analysis; Tapetum

\section{INTRODUCTION}

Male sterility (MS) occurs in plants when the female organ is normal but the male organ is abnormal and is unable to produce pollen or successfully pollinate. This is a common phenomenon in the plant kingdom and is important for both plant reproductive biology and crop-breeding research because it promotes heterosis or outbreeding (Wu et al., 2015). Kaul (1988) reported that MS had been observed in 43 families, 162 genera, 320 species, and 617 varieties or hybrids. According to the stage at which abortion occurs during anther development, MS can be classified into several types, including degradation of pollen mother cells, degeneration of tapetum cells, critical chemical changes in pollen wall development, and failure of anther dehiscence (Liu et al., 2014). For the promotion of heterosis, it is critical to obtain MS lines that interrupt the development of the anther and/or pollen (Zhang et al., 2006).

Plant anther tapetal cells comprise the interior layer of cells adjacent to the pollen mother cell in the anther clinandrium. The tapetum is of great importance for pollen development, and any process that blocks tapetal development will lead to MS. The tapetum has been studied in many plants using MS strains (Ma et al., 2015). Tapetal cells are essential for the formation of functional pollen grains; therefore, many MS mutations have been acquired through interference with tapetal cell differentiation and/or function degeneration (Li et al., 2015). Many studies have identified tapetum specific (TS) genes by screening MS mutants in Arabidopsis thaliana (Honys and Twell, 2003; Hsu et al., 2014) and transgenic MS plants have been produced through the degradation of tapetal cells.

Model plants are useful for translating genomic information between species. This cross-species translation is largely based on orthologous genes, which share a common evolutionary origin (Hyung et al., 2014). A. thaliana was the first plant species to have its genome fully sequenced, and among all model plants, the most comprehensive genomic studies have been performed on this species. Medicago truncatula is a model legume with a relatively small genome and short life cycle. It therefore serves as a central model system for translational genomics based studies in plants (Town et al., 2006). Although many studies have specifically studied MS in A. thaliana, Brassica oleracea, and Oryza sativa (Honys and Twell, 2003; Qu et al., 2008; Ji et al., 2011; Zhang and Zhang, 2014), few studies have assessed MS in forage plants. In this study, we attempted to identify tapetum specific male sterility (TSMS) related genes in M. truncatula, via comparisons with $A$. thaliana, as a source of genomic information. Information obtained from $M$. truncatula may provide critical data for the breeding of new germplasms in M. sativa.

\section{MATERIAL AND METHODS}

\section{Relational database}

M. truncatula genome sequence data were downloaded from JCVI (Mt4.0v1, http:// 
www.jcvi.org/medicago/). Expression data for M. truncatula TSMS related genes were downloaded from the M. truncatula Gene Expression Atlas (http://mtgea.noble.org/v3/). Gene sequence alignments and promoter analyses were conducted using NCBI (http://www.ncbi. nlm.nih.gov/) and the PLACE (http://www.dna.affrc.go.jp/PLACE/) software.

\section{Genome-wide screening of TSMS related genes and identification of cross-family orthologous genes}

Available data sources were used to identify TSMS related genes, including published literature, gene expression profiles, and the Arabidopsis database (TAIR, http://www.arabidopsis. org). We used key words relevant to TSMS to identify related genes in the Arabidopsis database, and each resulting gene description was investigated to confirm its functional relevance to TSMS. Genes identified from databases were combined into a single Excel file and organized according to locus ID along with other related information, such as location, cDNA length, exon number, intron number, coding sequence (CDS) length, and annotation (Table 1).

To identify orthologous genes in $M$. truncatula from $A$. thaliana, we used NCBI's BLASTp homolog search tool (http://blast.ncbi.nlm.nih.gov/Blast.cgi?PAGE=Proteins). We considered the E-value $\leq 1 \mathrm{e}-10$, identity $\geq 30 \%$, coverage $\geq 70 \%$, and the cumulative total length of alignment when identifying orthology between pairwise aligned genes through visual inspection (Hsu et al., 2014).

\section{Gene structure and chromosomal location}

Protein domain structure for domains with the lowest E-values was analyzed by the SMART online tool (http://smart.embl-heidelberg.de/). Information regarding cDNA sequences and chromosomal location of genes was retrieved from NCBI (http://www.ncbi. nlm.nih.gov/), and the location of each TSMS gene was then marked by the Mapinspect software (http://mapinspect.apponic.com/) (Ma et al., 2013).

\section{Phylogenetic analysis}

For phylogenetic analysis, we used the Clustal W2 software for multiple alignment of TSMS protein sequences from M. truncatula and A. thaliana. MEGA5.0 was then used to create phylogenetic trees using the neighbor-joining algorithm and a Poisson-corrected model with gaps/missing data subject to pairwise deletion and 1000 bootstrap test replications (Honys and Twell, 2003).

\section{Cis-element analysis}

The PLACE database (http://www.dna.affrc.go.jp/PLACE/) was used to search for cis-elements in the promoter regions of homologous TSMS related genes in M. truncatula. We selected a 2000-bp promoter region upstream of the start codon of each gene to search for possible cis-elements (Manimaran et al., 2015).

\section{Gene expression analysis}

Gene expression data were downloaded from the M. truncatula Gene Expression Atlas 
of the Samuel Roberts Noble Foundation. Transcriptome data included the analysis of root, stem, leaf, vegetative bud, flower, pod, and seed-coat tissue under various stress conditions, including $\mathrm{NH}_{4}, \mathrm{NO}_{3}, \mathrm{~N}_{2}, 1-\mathrm{Naphthaleneacetic} \mathrm{acid} \mathrm{(NAA),} \mathrm{and} \mathrm{Phymatotrichum} \mathrm{infection.}$ Data on TSMS gene expression were downloaded using the $\mathrm{MeV}$ (Ver.4.6.1) software (http:// www.tm4.org/mev/), and were formatted as $\log 2$ fold-change and graphically presented in a heat-map using the Cimminer software (http://discover.nci.nih.gov/cimminer/oneMatrix.do) (Ma et al., 2013).

\section{RESULTS}

\section{Identification of TSMS related genes}

To identify the full complement of TSMS related genes in M. truncatula, known TSMS genes from A. thaliana and the BLASTp algorithm were used to search the M. truncatula genome for homologs. A total of 63 putative M. truncatula TSMS related genes were identified (Table 1). Gene structure analyses revealed that these genes were characterized by highly variable exon and intron structures: 4 of the putative TSMS related genes contained no introns, 27 genes contained one intron, 7 genes contained two introns, 4 genes contained three introns, 12 genes contained four introns, 3 genes contained five introns, and 6 genes contained six or more introns. As intron patterns can shed light on phylogenetic relationships (Liu et al., 2014), these patterns indicate a significant degree of divergence among the putative TSMS genes (Ma et al., 2015). These 63 putative TSMS genes are located on eight $M$. truncatula chromosomes, ranging from three genes on chromosome 3 to 17 genes on chromosome 4 (Figure 1). The longest cDNA nucleotide sequence was $11.6 \mathrm{~kb}$ and included 20 exons, while the shortest cDNA sequence was $384 \mathrm{bp}$, comprising only one exon (Table 1).

\section{Structure of tapetum specific male sterility related proteins in $M$. truncatula}

Using the online program SMART, we accessed information on the functional domains of $M$. truncatula TSMS genes. Fifteen conserved domains (AAA, AAI, BBOX, HLH, HSP, Knot1, Pept_C1, Pfam, REC, RING, S_TKc, SANT, SERPIN, transmembrane region, and $\mathrm{UBCc}$ ) were identified by sequence alignment and protein position, and were renamed MtTsms1-63, clusters of orthologous groups A, B, and C, representing the three groups of MtTsms genes identified by the phylogenetic tree (Table 2). Among these, the conserved motif encoding the TSMS domain was found within all 63 proteins. Six genes (MtTsms1-6) contained the AAA domain, five genes (MtTsms7-11) contained the AAI domain, 37 genes contained Pfam domains, three genes (MtTsms57-59) contained SANT domains, and two genes (MtTsms62-63) contained UBCc domains, with the final 10 genes containing additional domains.

The presence of the AAA domain defines the AAA family of proteins, which often perform chaperone-like functions to assist in the assembly, operation, or disassembly of proteins; these proteins exhibit high ATPase activity and oligomerization (Ye et al., 2015). The AAI domain is present in several crucial proteins involved in pollen development (Tian et al., 2009). Although other identified conserved domains such as Pfam and the transmembrane region have not previously been associated with MS, our results suggest that the functions of these genes require further exploration. 
Table 1. Tapetum specific male sterility related genes in Medicago truncatula.

\begin{tabular}{|c|c|c|c|c|c|c|c|c|}
\hline Arabidopsis thaliana & Medicago truncatula & E-value & Location & cDNA length & Exon number & Intron number & CDS length & \begin{tabular}{|l|l|} 
Annotation \\
\end{tabular} \\
\hline $\begin{array}{l}\mathrm{AT1G} 01280 \\
\end{array}$ & \begin{tabular}{|l|l|} 
Medtr7g087520 \\
\end{tabular} & 0 & $\begin{array}{ll}\operatorname{chr} 7: 34077176-34078819 \\
\end{array}$ & 1644 & 2 & 1 & 1566 & \begin{tabular}{|c|} 
cytochrome $\mathrm{P} 450$ family protein \\
\end{tabular} \\
\hline \multirow{3}{*}{$\begin{array}{l}\text { AT1G27040 } \\
\end{array}$} & \begin{tabular}{|l|} 
Medtrlg053315 \\
Medro 5033380
\end{tabular} & 0 & chr1:22378573-22379970 & 1398 & $\frac{1}{5}$ & 0 & 1398 & HXXXD-type acyl-transferase family pro \\
\hline & \begin{tabular}{|l|l|} 
Medrtr590038380 \\
Medrtg 107670 \\
\end{tabular} & $\frac{0}{0}$ & 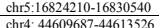 & $\frac{6331}{3840}$ & $\frac{5}{5}$ & $\frac{4}{4}$ & $\frac{1749}{1734}$ & \begin{tabular}{|l|} 
peptide//itratat transporter plant \\
peptidentitrate transporter
\end{tabular} \\
\hline & Medtr8g469310 & 0 & chr8:25169829-25178038 & 8210 & $\frac{5}{5}$ & 4 & 1734 & \begin{tabular}{|l|} 
peptider/mitrate transporter \\
peptide/nitrate transporter plant \\
\end{tabular} \\
\hline AT1G36340 & Medtr3g062450 & $1.00 \mathrm{E}-48$ & chr3:28197947-28201491 & 3545 & 4 & 3 & 447 & ubiquitin-conjugating enzyme E2 \\
\hline AT1G52560 & \begin{tabular}{|l|l|} 
Medtr4g010320.1 \\
\end{tabular} & $2.00 \mathrm{E}-57$ & $\begin{array}{ll}\text { chr4:2335470-2337224 } \\
\end{array}$ & 1755 & 2 & 1 & 696 & hsp20/alpha crystallin family protein \\
\hline AT1G62940 & Medtr2g105570 & $4.00 \mathrm{E}-139$ & chr2:45520733-45525707 & 4975 & 5 & 4 & 1656 & 4-coumarate:CoA ligase-like protein \\
\hline AT1G74310 & \begin{tabular}{|l|l|} 
Medtr4g109450 \\
\end{tabular} & & 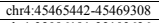 & 3867 & & 4 & 2739 & ATP-dependent chaperone ClpB \\
\hline AT1G06170 & \begin{tabular}{|l|l} 
Medtrl g072320 \\
\end{tabular} & $5.00 \mathrm{E}-10$ & chrl:32096191-32103626 & 7436 & 6 & 5 & 1971 & bHLH transcription factor \\
\hline $\begin{array}{l}\text { AT1G26780 } \\
A T 1616170\end{array}$ & Medtr4g082040 & $3.00 \mathrm{E}-74$ & $\begin{array}{l}\text { chr4:31789875-31791702 } \\
\end{array}$ & 1828 & 3 & 2 & 1284 & myb transcription factor \\
\hline $\begin{array}{l}\text { AT1G61070 } \\
\text { ATG06260 }\end{array}$ & \begin{tabular}{|l|l|} 
Medtr8g095375 \\
Medrt40079440
\end{tabular} & $\begin{array}{ll}3.00 \mathrm{E}-21 \\
1.0 \mathrm{E}-130\end{array}$ & 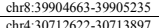 & 573 & $\frac{2}{2}$ & 1 & $\frac{225}{1038}$ & \begin{tabular}{|l|} 
defensin MtDef4.7 \\
\end{tabular} \\
\hline $\begin{array}{l}\text { AT1G0060 } \\
\text { AT1G0990 }\end{array}$ & \begin{tabular}{|l} 
Medrtag0 904440 \\
Medr5 g074270 \\
\end{tabular} & $\frac{1.00 \mathrm{E}-130}{1.00 \mathrm{E}-115}$ & $\begin{array}{l}\text { chr45.30/26222-30/1389/7 } \\
\text { chr5:31527639-31530478 }\end{array}$ & $\frac{1276}{2840}$ & $\frac{2}{4}$ & $\frac{1}{3}$ & $\frac{1038}{1101}$ & \begin{tabular}{|l} 
papann family y cystene protease \\
GDSL-like lipase/acylhydrolase \\
\end{tabular} \\
\hline AT1G09550 & Medtr7g111280 & $2.00 \mathrm{E}-158$ & chr7:45666166-45669433 & 3268 & 11 & 10 & 1260 & \begin{tabular}{|l} 
pectin acetylesterase family protein \\
\end{tabular} \\
\hline AT1G61110 & \begin{tabular}{|l|l|} 
Medtr2g080010 \\
\end{tabular} & $1.00 \mathrm{E}-108$ & chr2:337661666-33764021 & 2356 & 3 & 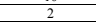 & 1065 & NAC transcription factor-like protetin \\
\hline \multirow[t]{3}{*}{$\begin{array}{l}\text { AT1G66570 } \\
\end{array}$} & \begin{tabular}{|l|} 
Medtrlg096910 \\
\end{tabular} & 0 & chrl $143663044-43666502$ & 3459 & 4 & 3 & 1578 & sucrose proton symporter, putative \\
\hline & \begin{tabular}{|l|l|} 
Medtr4g131920 \\
\end{tabular} & 0 & 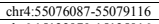 & 3030 & 5 & 4 & 1536 & sucrose transporter 2 \\
\hline & \begin{tabular}{|l|l} 
Medtr6g043880 \\
\end{tabular} & 0 & chr6:15122970-15125916 & 2974 & 5 & 4 & 1527 & sucrose transporter \\
\hline AT1G68190 & \begin{tabular}{|l|l} 
Medtr3g082630 \\
\end{tabular} & $7.00 \mathrm{E}-29$ & chr3:37271508-37275432 & 3925 & 6 & 5 & 1236 & B-box type zinc finger protein \\
\hline $\begin{array}{l}\text { AT1G71160 } \\
\text { ATG74450 }\end{array}$ & 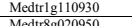 & $8.00 \mathrm{E}-162$ & 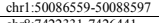 & 2039 & $\frac{1}{2}$ & 1 & 1491 & 3-ketoacyl-CoA synthase-like protein \\
\hline $\begin{array}{l}\text { AT1G74540 } \\
\text { ATG75930 }\end{array}$ & 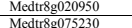 & $\begin{array}{l}1.00 \mathrm{E}-95 \\
800-74\end{array}$ & 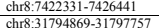 & 4111 & $\frac{2}{5}$ & $\frac{1}{4}$ & 1545 & 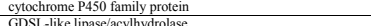 \\
\hline AT1G30860 & \begin{tabular}{|l} 
Medrtigg $0 / 5250$ \\
Medrl 090803 \\
\end{tabular} & $\frac{8.000-14}{3.00 \mathrm{E}-22}$ & 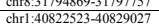 & $\frac{2889}{6505}$ & $\frac{3}{8}$ & $\frac{4}{7}$ & $\frac{1077}{2556}$ & \begin{tabular}{|l} 
GDSS-ilek lpaseracylihydrolase \\
RING/U-box protein
\end{tabular} \\
\hline AT2G16910 & \begin{tabular}{|l|} 
Medtr7g083900 \\
\end{tabular} & $2.00 \mathrm{E}-28$ & $\operatorname{chr} 7: 32316153-32319262$ & 3027 & 4 & 3 & 1428 & basic helix loop helix protein BHLH23 \\
\hline $\begin{array}{l}\text { AT2G14540 } \\
\end{array}$ & \begin{tabular}{|l|l|} 
Medtr7 7451890 \\
\end{tabular} & $2.00 \mathrm{E}-106$ & chr7:17697421-17699702 & 2282 & 2 & 1 & 1073 & \begin{tabular}{|l|} 
serpin-like protein \\
\end{tabular} \\
\hline AT2G26150 & \begin{tabular}{|l|l|} 
Medtr3g104550 \\
\end{tabular} & $3.00 \mathrm{E}-119$ & chr3:48170509-48171887 & 1379 & 2 & 1 & 1137 & heat shock transcription factor $\mathrm{A} 3$ \\
\hline AT2G31210 & \begin{tabular}{|l|l|} 
Medtr8g024790 \\
\end{tabular} & $6.00 \mathrm{E}-12$ & $\begin{array}{ll}\text { chr8:9212700-9214341 } \\
\end{array}$ & 1642 & 1 & 1 & 1395 & basic helix loop helix (bHLH) family transcription factor \\
\hline AT2G38240 & Medtr7g068650 & $2.00 \mathrm{E}-156$ & $\begin{array}{ll}\operatorname{chr} 7: 25175337-25179838 \\
\end{array}$ & 4502 & 3 & 2 & 1095 & leucoanthocyanidin dioxygenase-like protein \\
\hline $\begin{array}{l}\mathrm{AT} 2 \mathrm{G} 42940 \\
\mathrm{AT} \mathrm{G} 45630\end{array}$ & 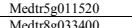 & $3.00 \mathrm{E}-101$ & chr5:39959479-39960243 & 765 & $\frac{1}{2}$ & 0 & 765 & AT hook motif DNA-binding family protein \\
\hline$\frac{\mathrm{AT} 2 \mathrm{G} 45630}{\mathrm{AT} 2 \mathrm{G} 0350}$ & \begin{tabular}{|l|l|} 
Medtrg80033400 \\
Medrtr2008610
\end{tabular} & $2.00 \mathrm{E}-115$ & chr8:12885180-12886877 & 1698 & 2 & $\frac{1}{2}$ & 1002 & D-isomer specific 2-hydroxyacid dehydrogenass domain prote \\
\hline$\frac{\mathrm{A} 12 \mathrm{O} 03030}{\mathrm{AT} 2 \mathrm{G} 2800}$ & \begin{tabular}{|l|l} 
Medtr8g078070 \\
\end{tabular} & $3.00 \mathrm{E}-140$ & $\begin{array}{l}\cos 2: 1532903-1535469 \\
\operatorname{chr} 8: 33275574-33277440\end{array}$ & $\frac{1520}{1867}$ & $\frac{1}{1}$ & $\frac{2}{1}$ & $\frac{786}{1119}$ & 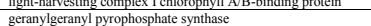 \\
\hline AT2G47040 & \begin{tabular}{|l|l|} 
Medtr7g110490 \\
\end{tabular} & $2.00 \mathrm{E}-129$ & chr7:45293122-45295123 & 2002 & 2 & 1 & 1731 & pectinesterase inhibitor domain protein \\
\hline AT3G05780 & Medtrlg084020 & 0 & chrl1:37455374-37466754 & 11381 & 20 & 19 & 2934 & Lon-related ATP-dependent protease, putative \\
\hline AT3G09640 & \begin{tabular}{|l|l|} 
Medtr4g061140 \\
\end{tabular} & $1.00 \mathrm{E}-146$ & chr4:22580311-22583517 & 3615 & 14 & 9 & 753 & \begin{tabular}{|l} 
L-ascorbate peroxidase \\
\end{tabular} \\
\hline AT3G13220 & Medtrl g054935 & 0 & $\begin{array}{cc}\operatorname{chr} 1: 24118450-24130074 \\
\end{array}$ & 11625 & 11 & 10 & 2229 & white-brown-complex $\mathrm{ABC}$ transporter family protein \\
\hline \multirow[t]{2}{*}{$\begin{array}{l}\text { AT3G48540 } \\
\end{array}$} & 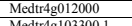 & $1.00 \mathrm{E}-10$ & $\begin{array}{ll}\text { chr4:3190971-3199290 } \\
\end{array}$ & 8320 & 6 & 5 & 3747 & tRNA-specific adenosine deaminase \\
\hline & \begin{tabular}{|l|l|} 
Medtr4g103300.1 \\
Medtro065160
\end{tabular} & $8.00 \mathrm{E}-122$ & chr4:42684135-42689049 & 4915 & $\frac{9}{2}$ & 8 & 699 & tRNA-specific adenosine deaminase \\
\hline$\frac{\mathrm{A} 13 \mathrm{G} 52130}{\mathrm{AT} 3 \mathrm{G} 13890}$ & \begin{tabular}{|l|} 
Medtr7g0605160 \\
Medrog 09740 \\
\end{tabular} & $\frac{1.000-10}{4000-78}$ & chr7:237544468-237755721 & $\frac{1254}{1804}$ & $\frac{2}{2}$ & 1 & $\frac{354}{1206}$ & lipid transfer protein \\
\hline AT3G51590 & \begin{tabular}{|l|l|l|l} 
Medtr4g027800 \\
\end{tabular} & $2.00 \mathrm{E}-28$ & chrt4:98111604-98412898 & 1205 & $\frac{2}{2}$ & 1 & $\frac{1206}{351}$ & \begin{tabular}{|l|} 
lipid transfer protein \\
\end{tabular} \\
\hline AT3G56380 & Medtr5g036480 & $4.00 \mathrm{E}-54$ & chr5:15918294-15920037 & 1744 & 5 & 4 & 714 & two-component response regulator ARR3-like protein \\
\hline $\begin{array}{l}\text { AT4G14080 } \\
\end{array}$ & \begin{tabular}{|l|l|} 
Medtr7g053500 \\
\end{tabular} & 0 & chr7:18857464-18859936 & 2473 & 3 & 2 & 1392 & glucan endo-1,3-beta-glucosidase-like protein \\
\hline \multirow[t]{3}{*}{$\begin{array}{l}\text { AT4G20800 } \\
\end{array}$} & \begin{tabular}{|l|l|} 
Medtr4g091200 \\
\end{tabular} & 0 & $\begin{array}{cc}\text { chr4:36107336-36109152 } \\
\end{array}$ & 1817 & 1 & 1 & 1587 & FAD-binding berberine family protein \\
\hline & \begin{tabular}{|l|l|} 
Medtrog0017205 \\
\end{tabular} & 0 & $\begin{array}{ll}\text { chr6:6838164-6839789 } \\
\end{array}$ & 1626 & 1 & 0 & 1626 & FAD-binding berberine family protein \\
\hline & Medtr- & 0 & chr4:34970644 & 1620 & 1 & 0 & 1620 & \begin{tabular}{|l} 
FAD-binding berberine family protein \\
\end{tabular} \\
\hline \multirow{3}{*}{$\begin{array}{l}\mathrm{ATSG} 02490 \\
\end{array}$} & 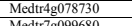 & 0 & chr4:304: & 1282 & $\frac{2}{2}$ & 1 & 1170 & chalcone synthase family protein, putative \\
\hline & \begin{tabular}{|l|l|} 
Medtr7g099680 \\
Medrfo 02450 \\
\end{tabular} & $\frac{0}{0}$ & chr7:39984070-39987331 & 3262 & $\frac{2}{2}$ & 1 & 1950 & \begin{tabular}{|l|l|} 
heat shock cognate $70 \mathrm{kDa}$ protein \\
hat sheck
\end{tabular} \\
\hline & 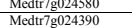 & $\frac{0}{0}$ & 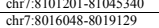 & $\frac{3334}{3382}$ & $\frac{2}{2}$ & $\frac{1}{1}$ & $\frac{1950}{1947}$ & $\begin{array}{l}\text { heat shock coggate } 70 \mathrm{kDa} \text { p protein } \\
\text { heat shock cognate } 70 \mathrm{kDa} \text { protein }\end{array}$ \\
\hline $\begin{array}{l}\text { AT5G09970 } \\
\end{array}$ & Medtrgg027040 & $5.00 \mathrm{E}-156$ & chr8:9421677-9424147 & 2471 & 2 & 1 & 1641 & cytochrome $\mathrm{P} 450$ family 78 protein \\
\hline AT5G59720 & Medtrog061940 & $6.00 \mathrm{E}-83$ & chr6:24040583-24041280 & 698 & 1 & 1 & 480 & $17.6 \mathrm{kDa}$ class I heat shock protein \\
\hline AT5G03800 & Medtr7g103230 & 0 & chr7:41735375-41738881 & 3507 & 3 & 2 & 2913 & PPR containing plant-like protein \\
\hline $\begin{array}{l}\text { AT5G07230 } \\
\end{array}$ & Medtr 1 & $2.00 \mathrm{E}-18$ & chrl:45783546-45783929 & 384 & 2 & 1 & 294 & lipid transfer protein (LIM1/2/3/MEN-8) \\
\hline \multirow[t]{3}{*}{$\begin{array}{l}\text { ATSG15250 } \\
\end{array}$} & \begin{tabular}{|l|l|l} 
Medtr7g010800 \\
\end{tabular} & 0 & $\begin{array}{ll}\operatorname{chr} 7: 2723939-2728362 \\
\end{array}$ & 4424 & 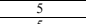 & 4 & 2014 & \begin{tabular}{|l|l|l|l|l} 
ATP-dependent zinc metallopotease FTSH protein \\
STSS
\end{tabular} \\
\hline & \begin{tabular}{|l|l} 
Medtr2g438140 \\
\end{tabular} & 0 & chr2:15376886-15381581 & 4696 & $\frac{5}{5}$ & 4 & 2073 & ATP-dependent zinc metalloprotease FTSH protein \\
\hline & \begin{tabular}{|l|} 
Medrtug0 094662 \\
Medrto 009430 \\
\end{tabular} & 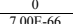 & chr4:38677988-38681608 & $\frac{3621}{2228}$ & 3 & $\frac{4}{2}$ & 2085 & ATP-dependent zinc metalloprotease FTSH protein \\
\hline AT5G59330 & \begin{tabular}{|l} 
Medirggo 09430 \\
Medrtg 027840 \\
\end{tabular} & $1.00 \mathrm{E}-66$ & $\begin{array}{l}\text { chro: } 2 / 90201-2 / 942486 \\
\text { chr4:9831935-9832433 }\end{array}$ & $\frac{2286}{499}$ & $\frac{3}{2}$ & $\frac{2}{1}$ & $\frac{101}{420}$ & \begin{tabular}{|l|} 
mybo transscription Iact \\
lipid transfer protein
\end{tabular} \\
\hline AT5G66110 & \begin{tabular}{|l|l} 
Medtr4g073040 \\
\end{tabular} & $6.00 \mathrm{E}-72$ & chr4:27630362-27631603 & 1242 & 2 & 1 & 471 & heavy metal transport/detoxification superfamily protein \\
\hline AT5G14980 & \begin{tabular}{|l|l} 
Medtrl 0095110 \\
\end{tabular} & $9.00 \mathrm{E}-166$ & chrl:42811469-42812783 & 1315 & 1 & 1 & 978 & alpha/beta hydrolase family protein \\
\hline AT5G38160 & \begin{tabular}{|l|l} 
Medtrog0 082480 \\
\end{tabular} & $9.00 \mathrm{E}-19$ & chr6:30850320-30851288 & 969 & 1 & 1 & 279 & chitinase/hevein/PR-4/wheatwin2 \\
\hline AT5G57670 & \begin{tabular}{|l|} 
Medrtyg128990 \\
\end{tabular} & 0 & chr4:53672087-53675818 & 3732 & 10 & 9 & 1728 & receptor-like kinase \\
\hline
\end{tabular}

CDS means coding sequence.

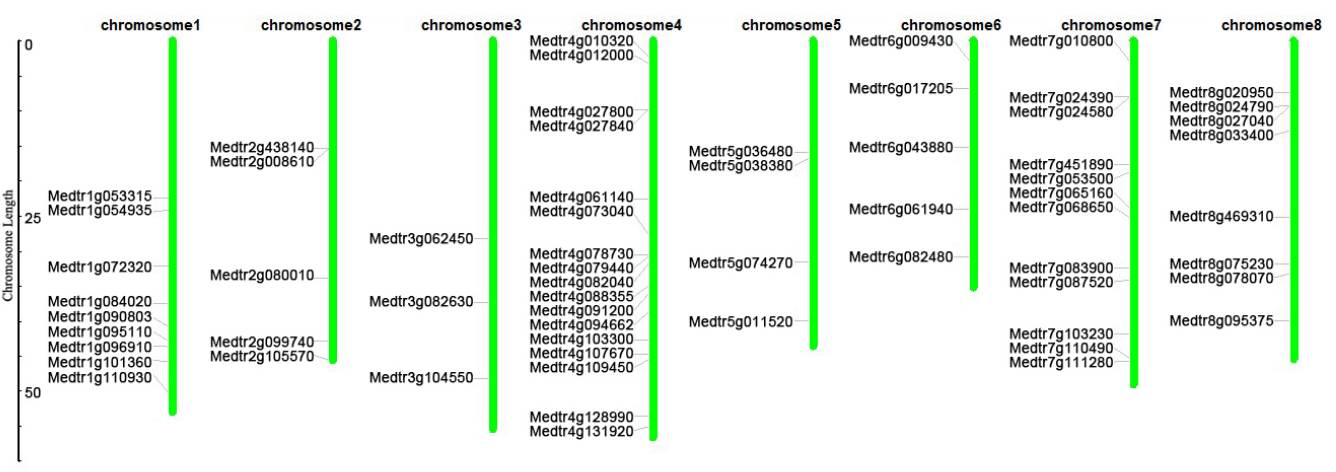

cM

Figure 1. Chromosomal locations of tapetum specific male sterility related genes in Medicago truncatula. 
Table 2. Conserved domains in tapetum specific male sterility related genes of Medicago truncatula.

\begin{tabular}{|c|c|c|c|c|c|c|}
\hline Gene name & Gene ID & Predicted domains & Clusters of orthologous groups & Start & End & E-value \\
\hline MtTsms1 & Medtrlg084020 & AAA & $\mathrm{B}$ & 458 & 603 & $7.54 \mathrm{e}-12$ \\
\hline MtTsms2 & Medtrlg054935 & AAA & $\mathrm{B}$ & 19 & 210 & $5.41 \mathrm{e}-13$ \\
\hline MtTsms3 & Medtr2g438140 & AAA & $\mathrm{C}$ & 261 & 400 & $6.06 \mathrm{e}-25$ \\
\hline MtTsms4 & Medtr4g094662 & AAA & $\mathrm{C}$ & 264 & 403 & $4.28 \mathrm{e}-25$ \\
\hline MtTsms5 & Medtr4g109450 & AAA & $\mathrm{A}$ & 601 & 772 & $8.08 \mathrm{e}-12$ \\
\hline MtTsms6 & Medtr7g010800 & AAA & $\mathrm{C}$ & 241 & 380 & $8.7 \mathrm{e}-26$ \\
\hline MtTsms7 & Medtr1g101360 & AAI & $\mathrm{C}$ & 34 & 94 & $1.14 \mathrm{e}-8$ \\
\hline MtTsms8 & Medtr4g027840 & AAI & $\mathrm{A}$ & 27 & 111 & $6.77 \mathrm{e}-16$ \\
\hline MtTsms9 & Medtr4g027800 & AAI & $\mathrm{A}$ & 27 & 111 & $6.77 \mathrm{e}-16$ \\
\hline MtTsms 10 & Medtr6g082480 & AAI & A & 27 & 92 & 0.0013 \\
\hline MtTsms11 & Medtr7g065160 & AAI & $\mathrm{C}$ & 29 & 111 & 0.00000614 \\
\hline MtTsms 12 & Medtr3g082630 & BBOX & $\mathrm{C}$ & 4 & 47 & $1.5 \mathrm{e}-8$ \\
\hline MtTsms 13 & Medtr7g083900 & HLH & A & 288 & 337 & N/A \\
\hline MtTsms14 & Medtr3g104550 & HSF & $\mathrm{C}$ & 43 & 136 & $2.65 \mathrm{e}-58$ \\
\hline MtTsms15 & Medtr8g095375 & Knot1 & A & 29 & 74 & $6.37 \mathrm{e}-11$ \\
\hline MtTsms16 & Medtr4g079440 & Pept $\mathrm{Cl}$ & A & 128 & 344 & $6.58 \mathrm{e}-118$ \\
\hline MtTsms17 & Medtr8g033400 & Pfam:2-Hacid_dh_c & $\mathrm{C}$ & 57 & 333 & $8.9 \mathrm{e}-19$ \\
\hline MtTsms 18 & Medtr1 g095110 & Pfam:Abhydrolase 5 & $\mathrm{~B}$ & 54 & 298 & $2.7 \mathrm{e}-56$ \\
\hline MtTsms 19 & Medtr2g105570 & Pfam:AMP-binging & $\mathrm{C}$ & 32 & 451 & $1.5 \mathrm{e}-107$ \\
\hline MtTsms20 & Medtr8g024790 & Pfam:Bhlh-MYC N & $\mathrm{B}$ & 26 & 181 & $4.5 \mathrm{e}-41$ \\
\hline MtTsms21 & Medtrlg072320 & Pfam:Bhlh-MYC N & $\mathrm{C}$ & 11 & 188 & $2.9 \mathrm{e}-56$ \\
\hline MtTsms 22 & Medtr2g008610 & Pfam:Chloroa b-bind & $\mathrm{C}$ & 72 & 250 & $4.2 \mathrm{e}-48$ \\
\hline MtTsms23 & Medtr4g012000 & Pfam:dCMP cyt & A & 1045 & 1146 & $3.7 \mathrm{e}-26$ \\
\hline MtTsms24 & Medtr7g068650 & Pfam:DIOX N & A & 54 & 166 & $1.4 \mathrm{e}-31$ \\
\hline MtTsms25 & Medtr5g011520 & Pfam:DUF296 & $\mathrm{C}$ & 128 & 241 & $1.2 \mathrm{e}-30$ \\
\hline MtTsms26 & Medtr7g103230 & Pfam:DYW deaminase & $\mathrm{C}$ & 741 & 865 & $2.3 e-33$ \\
\hline MtTsms27 & Medtr4g091200 & Pfam:FAD binding4 & $\mathrm{C}$ & 74 & 212 & $1.4 \mathrm{e}-27$ \\
\hline MtTsms28 & Medtr4g088355 & Pfam:FAD binding 4 & A & 81 & 219 & $1.7 \mathrm{e}-25$ \\
\hline MtTsms 29 & Medtr6g017205 & Pfam:FAD binding 4 & A & 74 & 212 & $2 \mathrm{e}-26$ \\
\hline MtTsms30 & Medtr4g078730 & Pfam:FAE_CUT1_RppA & A & 81 & 260 & $4.5 \mathrm{e}-8$ \\
\hline MtTsms31 & Medtr7g053500 & Pfam:Glyco hydro 17 & A & 26 & 348 & $7.9 \mathrm{e}-102$ \\
\hline MtTsms32 & Medtr4g073040 & Pfam:HMA & $\mathrm{C}$ & 31 & 89 & $1.6 \mathrm{e}-16$ \\
\hline MtTsms 33 & Medtr4g010320 & Pfam:HSP20 & $\mathrm{C}$ & 125 & 231 & $4.2 \mathrm{e}-26$ \\
\hline MtTsms34 & Medtr6g061940 & Pfam:HSP20 & $\mathrm{C}$ & 55 & 158 & $7.5 e-35$ \\
\hline MtTsms35 & Medtr7g099680 & Pfam:HSP70 & A & 9 & 618 & $6.5 \mathrm{e}-269$ \\
\hline MtTsms36 & Medtr7g024390 & Pfam:HSP70 & A & 9 & 618 & $1.9 \mathrm{e}-269$ \\
\hline MtTsms 37 & Medtr7g024580 & Pfam:HSP70 & A & 9 & 618 & $1.9 \mathrm{e}-269$ \\
\hline MtTsms38 & Medtr5g074270 & Pfam:Lipase GDSL & A & 34 & 348 & $1.7 \mathrm{e}-20$ \\
\hline MtTsms39 & Medtr8g075230 & Pfam:Lipase GDSL & A & 37 & 349 & $6.6 \mathrm{e}-13$ \\
\hline MtTsms40 & Medtrlg096910 & Pfam:MFS 2 & A & 37 & 521 & $2 \mathrm{e}-9$ \\
\hline MtTsms41 & Medtr2g080010 & Pfam:NAM & $\mathrm{C}$ & 16 & 142 & $3.3 \mathrm{e}-33$ \\
\hline MtTsms42 & Medtr8g027040 & Pfam:p450 & $\mathrm{C}$ & 85 & 532 & $4.1 \mathrm{e}-82$ \\
\hline MtTsms 43 & Medtr8g020950 & Pfam:p450 & A & 39 & 500 & $7.4 \mathrm{e}-97$ \\
\hline MtTsms44 & Medtr7g087520 & Pfam:p450 & A & 38 & 509 & $1.6 \mathrm{e}-96$ \\
\hline MtTsms45 & Medtr7g111280 & Pfam:PAE & $\mathrm{B}$ & 50 & 400 & $2.9 \mathrm{e}-164$ \\
\hline MtTsms46 & Medtr7g110490 & Pfam:Pectinesterase & A & 258 & 555 & $1.7 \mathrm{e}-122$ \\
\hline MtTsms47 & Medtr4g061140 & Pfam:peroxidase & $\mathrm{C}$ & 8 & 227 & $5.4 \mathrm{e}-47$ \\
\hline MtTsms48 & Medtr8g078070 & Pfam:polyprenyl synt & $\mathrm{C}$ & 110 & 357 & $3.4 \mathrm{e}-58$ \\
\hline MtTsms49 & Medtr5g038380 & Pfam:PTR2 & A & 100 & 536 & $1.7 \mathrm{e}-109$ \\
\hline MtTsms50 & Medtr4g107670 & Pfam:PTR2 & $\mathrm{B}$ & 96 & 533 & $7 \mathrm{e}-105$ \\
\hline MtTsms51 & Medtr8g469310 & Pfam:PTR2 & A & 98 & 533 & $7 \mathrm{e}-105$ \\
\hline MtTsms52 & Medtr3g098980 & Pfam:Transferase & A & 12 & 433 & $8.8 \mathrm{e}-93$ \\
\hline MtTsms53 & Medtrlg053315 & Pfam:Transferase & A & 16 & 457 & $1.8 \mathrm{e}-75$ \\
\hline MtTsms54 & Medtr5g036480 & REC & $\mathrm{B}$ & 16 & 147 & $2.27 \mathrm{e}-18$ \\
\hline MtTsms55 & Medtrlg090803 & RING & $\mathrm{B}$ & 799 & 837 & 0.249 \\
\hline MtTsms56 & Medtr4g128990 & S TKc & $\mathrm{C}$ & 262 & 531 & $6.27 \mathrm{e}-16$ \\
\hline MtTsms57 & Medtr6g009430 & SANT & $\mathrm{B}$ & 13 & 63 & $8.69 \mathrm{e}-14$ \\
\hline MtTsms58 & Medtr4g082040 & SANT & $\mathrm{C}$ & 10 & 21 & N/A \\
\hline MtTsms59 & Medtr2g099740 & SANT & $\mathrm{B}$ & 13 & 63 & $4.92 \mathrm{e}-13$ \\
\hline MtTsms60 & Medtr7g451890 & SERPIN & $\mathrm{C}$ & 24 & 399 & $2.93 \mathrm{e}-90$ \\
\hline MtTsms61 & Medtr4g131920 & transmembrane region & $\mathrm{B}$ & 37 & 56 & N/A \\
\hline MtTsms62 & Medtr3g062450 & UBCc & A & 4 & 147 & $5.22 \mathrm{e}-81$ \\
\hline MtTsms63 & Medtrlg110930 & $\mathrm{UBCc}$ & A & 4 & 147 & $5.22 \mathrm{e}-81$ \\
\hline
\end{tabular}

\section{Phylogeny of TSMS related genes in M. truncatula}

In order to compare the potential functions of TSMS related genes in M. truncatula and A. thaliana, we conducted a phylogenetic analysis with all 63 genes from M. truncatula 
and 53 homologous genes from A. thaliana. Based on phylogenetic relationships and domain organization, genes could be separated into three groups (MtTsms A, MtTsms B, and MtTsms C), each of which contains genes encoding proteins with the same or similar domain organizations, as shown in Figure 2. A total of 28 TSMS genes from M. truncatula and 17 TSMS genes from A. thaliana were included in MtTsms A, while 11 TSMS genes from $M$. truncatula and 16 TSMS genes from A. thaliana were included in MtTsms B, and MtTsms C contained 24 TSMS genes from $M$. truncatula and 20 TSMS genes from A. thaliana. These results indicate that there is high homology among most TSMS related genes in $M$. truncatula. Interestingly, some proteins carrying the same domain were occasionally separated into different groups, such as MtTsms1 and 2; MtTsms5, 7, and 11; and MtTsms8, 9, and 10 (Table 2). This implies that the evolutionary histories of proteins with unknown C-terminal and $\mathrm{N}$-terminal domains may be complex.

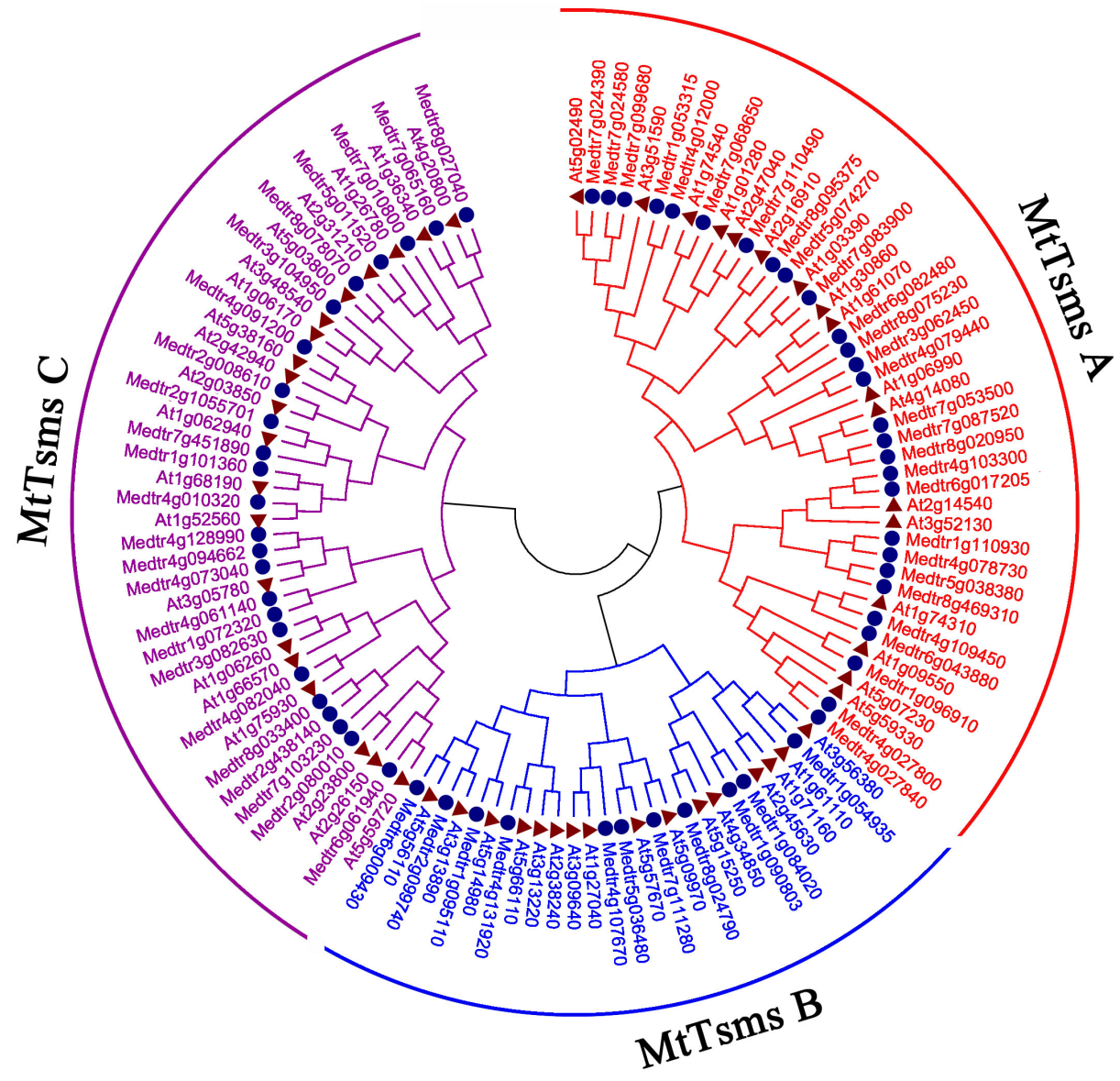

Figure 2. Neighbor-joining tree of tapetum specific male sterility related genes in Medicago truncatula and Arabidopsis thaliana. Tsms A genes are shown in red, Tsms B genes are shown in blue, and Tsms C genes are shown in purple. 


\section{Cis-acting regulatory elements of TSMS related genes in M. truncatula}

Promoters, which are comprised of various cis-regulatory elements, control the development and physiology of plants by regulating gene expression (Pauls et al., 2015). To identify cis-regulatory elements that potentially mediate the transcription of TSMS genes, promoter regions of the TSMS genes were analyzed using the PLACE database. In this study, eight cis-elements (ABRELATERD1, ARR1AT, CAAT box, CACTFTPPCA1, GTGANTG10, POLLENLELAT52, TATA box, and WRKY71OS) were identified in the promoters of putative TSMS genes (Table 3). ABRELATERD1 is a hormonal-response element, while ARR1AT is involved in cytokinin responsiveness (Li et al., 2015). The CAAT box is generally found upstream of the TATA box; it can be combined with one or more transcription factors and may be related to the structure of RNA polymerase II (Van Opijnen et al., 2004). CACTFTPPCA1 enhances the expression of light reaction components (Wilson and Zhang, 2009). GTGANTG10 and POLLENLELAT52 are enhancer elements of LAT52 and LAT56, and are essential for high expression levels in pollen (Manimaran et al., 2015). The TATA box is an essential element of the eukaryotic promoter and controls the accuracy and frequency of transcription (Van Opijnen et al., 2004). WRKY71OS is involved in components of defense reactions and related disease-resistance (Zhang et al., 2004).

Table 3. Characteristics of cis-motifs of male sterility genes identified using the PLACE database.

\begin{tabular}{l|l|l|c}
\hline PLACE cis-motif & Sequence & Function & No. of genes \\
\hline ABRELATERD1 & ACGTG & hormone-responsive elements & 47 \\
\hline ARR1AT & NGATT & element involved in cytokinin responsiveness & 63 \\
\hline CAATBOX1 & CAAT & defense-responsive element & 63 \\
\hline CACTFTPPCA1 & YACT & light-responsive elements & 55 \\
\hline GTGANTG10 & GTGA & pollen & 62 \\
\hline POLLEN1LELAT52 & AGAAA & pollen & 62 \\
\hline TATABOX & TATAAAT & TATA box & 63 \\
\hline WRKY71OS & TGAC & defense-responsive element & 60 \\
\hline
\end{tabular}

\section{Expression profiles of TSMS related genes in M. truncatula}

To investigate the expression patterns of TSMS related genes in different tissues, RNA-seq data for 58 genes (all existing data) were downloaded from the M. truncatula Gene Expression Atlas of the Samuel Roberts Noble Foundation. Three-color clustering was used to indicate the strength of gene expression, with bright red representing the strongest signal, bright green representing the weakest signal, and black representing an average signal. The results (Figure 3) illustrated differential transcript abundances of the 58 genes in seven tissues, including root, stem, leaf, vegetative bud, flower, pod, and seed coat. Four genes (MtTsms7, 16, 30, and 57) were expressed at high levels across all seven tissues, indicating a role in constitutive transport processes throughout the plant (Paul et al., 1992). Nine genes (MtTsms5, $14,27,32,33,34,43,49$, and 51) were found to be expressed at low levels across all tissues. The expression profiles of these genes diverged, implying that they contribute to functional maintenance through regulatory subfunctionalization or neofunctionalization $(\mathrm{Qu}$ et al., 2008). MtTsms11 and MtTsms 25 were highly expressed in root tissue, while MtTsms 19 and MtTsms56 were highly expressed in stem tissue. MtTsms 2 was highly expressed in leaves, and MtTsms8, 9, 35, 46, 48, and 63 were highly expressed in flowers. To identify genes with 
potential roles in $M$. truncatula abiotic and biotic stress responses, the expression patterns of all 58 genes were investigated in plants exposed to high salinity, $\mathrm{NH}_{4}, \mathrm{NO}_{3}, \mathrm{~N}_{2}, \mathrm{NAA}$, and Phymatotrichum infection. MtTsms19, 20, 27, 32, 40, 49, and 52 were upregulated in root tissues under salt stress, indicating that these genes may be involved in the salt stress response. MtTsms2, 3, 13, 63, and 48 were upregulated in leaves in the presence of the growth regulator NAA, indicating that these genes may be involved in plant growth associated with an NAA response. MtTsms 10, 11, 25, 43, and 47 were upregulated in roots, but downregulated in shoots under nitrogen stress. Only MtTsms47 was upregulated in roots under Phymatotrichum infection.

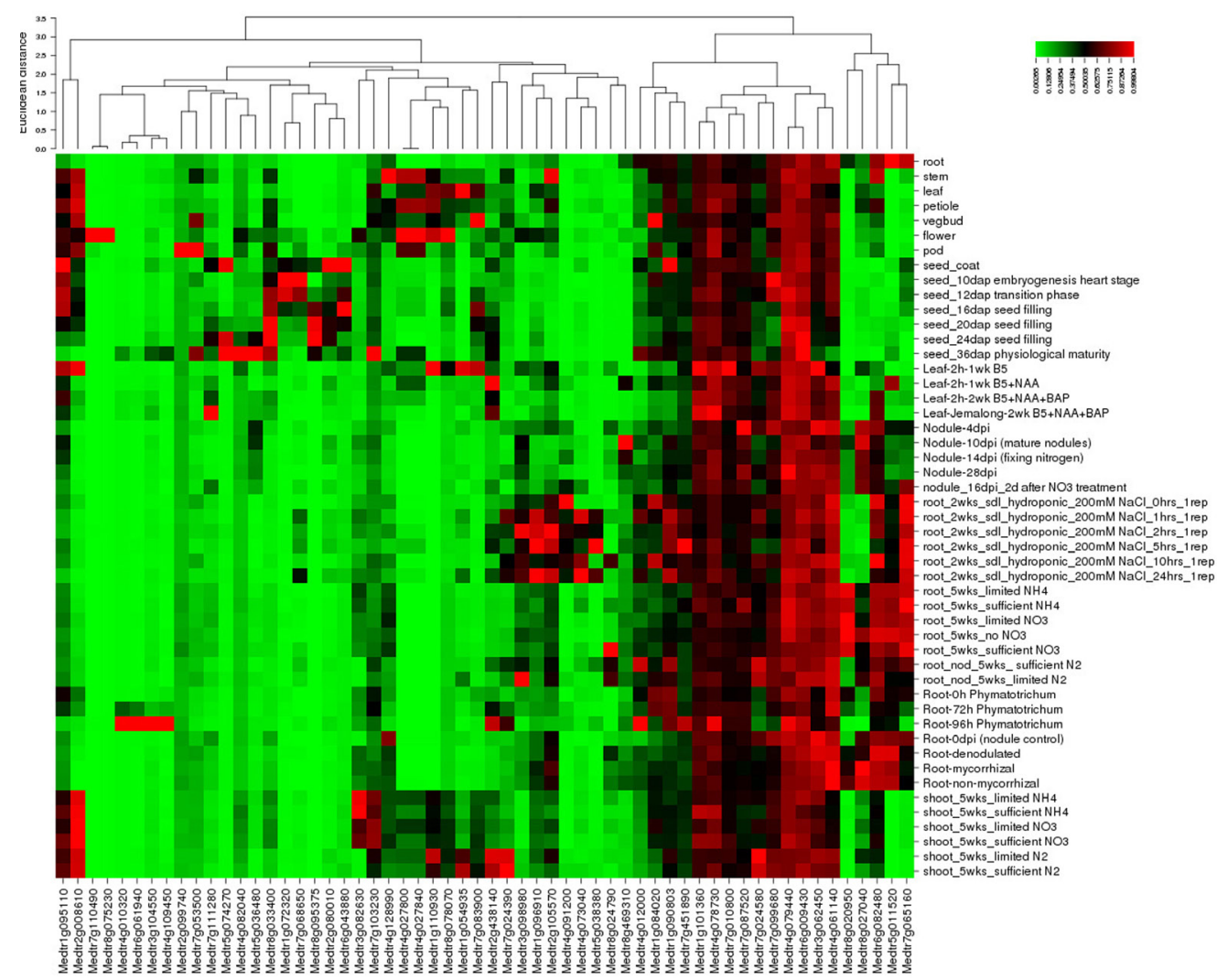

Figure 3. Expression profiles of TSMS related genes in Medicago truncatula. Three-color clustering indicates the strength of gene expression, with bright red representing the strongest signal, bright green representing the weakest signal, and black representing an average level of expression.

\section{DISCUSSION}

Following the determination of the Medicago and Arabidopsis genome sequences, data became available for the comparative study of MS related genes in the two plants. The results of this study may provide novel insights into the evolution and development of TSMS genes in M. truncatula. We identified 63 putative TSMS related genes in M. truncatula and 
investigated their structure and chromosomal organization. In previous studies, $P U B 4, A 6$, LTP12, LAP5, and TAP44 were identified in Arabidopsis and were found to play an important role in early tapetum formation and floral organ differentiation (Liu et al., 2009; Kim et al., 2010; Matsuo et al., 2013; Wang et al., 2013). In our study, homologs of these genes in $M$. truncatula were all found to contain a domain associated with sterility. In addition, a large percentage of TSMS related genes contained very few introns $(49.20 \%$ with $0-1$ intron), similar to those of $A$. thaliana and $O$. sativa (Zhang et al., 2006; Matsuo et al., 2013).

Based on a phylogenetic tree and the analysis of conserved domains, we found that Medicago and Arabidopsis genes clustered closely together, supporting the close evolutionary relationship of the two species (Liu et al., 2009). Both species are dicotyledonous rosids, a group that appeared 108-117 million years ago (Wikström et al., 2001). We found similar results when BLAST was used to identify $M$. truncatula homologs of $A$. thaliana TSMS related genes. Through analysis of conserved domains in the resulting proteins, we discovered that TSMS related genes often contain domains associated with sterility traits, including the AAA, AAI, BBOX, HLH, Knot1, RING, and SANT domains. The AAA domain confers ATPase activity, and correlates with abnormal development in plants (Ye et al., 2015). The AAI domain is found in the BcMF15 gene of B. oleracea, which is mainly expressed in the stamen, indicating that the AAI domain may participate in fertility regulation (Tian et al., 2009). The BBOX protein is involved in the development of light signal conditioning in $A$. thaliana (Kumagai et al., 2008). The HLH domain is found in the gene OsHLH164 and is expressed in the boundary region between the apical meristem and the new formative meristem; this gene is the main regulatory factor controlling the formation of axillary bud primordia in $O$. sativa and contributes to plant growth and development (Zhu et al., 2005). The defensin gene Bnfef of Brassica napus contains the Knot1 domain, which plays a significant role in the plant's defense response. The expression of the defensin gene is highest in flower buds, suggesting that it may also be related to fertility (Zheng et al., 2015). AtDUO1 and AtDUO3 from $A$. thaliana both contain SANT domains and participate in male reproductive cell division and differentiation, influencing sporophyte and male gamete development (Brownfield et al., 2009). REC and S_TKc domains are correlated with disease resistance. In a study of $O$. sativa, the REC domain was found to be associated with phosphoric acid signal recognition receptors, and the S_TKc domain was found to be associated with serine and threonine protein kinase family; genes containing these two domains are believed to be involved in disease resistance (Manimaran et al., 2015). UBCc, a ubiquitin combined with an enzyme E2 catalytic domain structure, was found in a cloned $\mathrm{ZmPHO} 2$ protein in inbred maize lines and is related to the dynamic balance of phosphorus in plants. The HSP domain is found in heat-shock factors, which are less common in plants (Pant et al., 2015). The binding site of the O. sativa gene OsHsp90 is located behind an ATPase domain and plays an important role in proteins related to resistance (Raman and Suguna, 2015). The Pept C1 domain has been extensively studied in animals, where it is expressed at the highest level in the kidneys (Funkelstein et al., 2008); however, it has not been previously reported in plants.

Although the function of most gene products is dependent on both transcriptional and post-transcriptional regulation, the spatial and temporal expression patterns and transcript levels of genes are primarily regulated by the cis-elements present in promoters (Qu et al., 2008). Analysis of the cis-elements present among M. truncatula TSMS related genes identified eight different cis-elements (ABRELATERD1, ARR1AT, CAATBOX1, CACTFTPPCA1, GTGANTG10, POLLEN1LELAT52, TATABOX, and WRKY71OS). In a 
previous study, ABRELATERD1 was found to be a hormone-responsive element related to the binding of MYB and zinc finger motif MADS-box transcription factors to promoter regions (Mahajan et al., 2015). ARR1AT, GTGANTG10, WRKY71OS, and TATABOX5 elements are strong candidates for transcriptional enhancers (Pauls et al., 2015). POLLEN1LELAT52 and GTGANTG10 are pollen-specific cis-elements, identified in bicellular and tricellular pollen enriched genes, respectively, among rice TSMS genes (Wei et al., 2010). The WRKY71OS element, which is highly represented among $M$. truncatula TSMS genes, contains an OsWRKY71 binding site, suggesting that WRKY71OS may participate in gibberellin signal repression (Zhang et al., 2004).

Our transcriptome analysis revealed that four genes (MtTsms7, 16, 30, and 57) were expressed among all seven tissues analyzed, while nine genes (MtTsms5, 14, 27, 32, 33, 34, 43,49 , and 51) were found to have low expression among all tissue types, and six genes (MtTsms8, 9, 35, 46, 48, and 63) demonstrated high expression in flowers. These patterns indicate that gene expression during different developmental stages can result in abnormal downstream or upstream reactions (Fujii et al., 2010), and that many genetic pathways are associated with the tapetum developmental network (Wilson and Zhang, 2009). Construction of a heat-map showing gene expression under various osmotic-related stresses in different tissues/organs (Figure 3 ) revealed that these genes play important roles in regulating the stress response (Qu et al., 2008). Taken together, our comparative analysis data of TSMS related genes showed that these genes vary in terms of their expression patterns in M. truncatula.

In summary, we identified 63 putative TSMS related genes in M. truncatula. We performed comprehensive analyses of gene structure, conserved domains, cis-elements, phylogeny, and expression profiling in various tissues, and under abiotic stresses. Based on structural characteristics and a comparison of the phylogenetic relationships between $M$. truncatula and $A$. thaliana homologs, the genes were classified into three groups. Conserved domains related to sterility (AAA, AAI, BBOX, HLH, Knot1, RING, and SANT) and pollenspecific cis-elements (GTGANTG10 and POLLEN1LELAT52) were identified. Expression analyses revealed that putative TSMS related genes may be involved in developmental processes and abiotic stress responses. This study provides an overview of these genes in M. truncatula and provides insights into MS, although the mechanisms underlying the participation of these genes require further study.

\section{Conflicts of interest}

The authors declare no conflict of interest.

\section{ACKNOWLEDGMENTS}

Research supported by the National Science Foundation of China (\#31372362).

\section{REFERENCES}

Brownfield L, Hafidh S, Durbarry A, Khatab H, et al. (2009). Arabidopsis DUO POLLEN3 is a key regulator of male germline development and embryogenesis. Plant Cell 21: 1940-1956. http://dx.doi.org/10.1105/tpc.109.066373

Fujii S, Yamada M, Fujita M, Itabashi E, et al. (2010). Cytoplasmic-nuclear genomic barriers in rice pollen development revealed by comparison of global gene expression profiles among five independent cytoplasmic male sterile lines. 
Plant Cell Physiol. 51: 610-620. http://dx.doi.org/10.1093/pcp/pcq026

Funkelstein L, Toneff T, Hwang SR, Reinheckel T, et al. (2008). Cathepsin L participates in the production of neuropeptide $\mathrm{Y}$ in secretory vesicles, demonstrated by protease gene knockout and expression. J. Neurochem. 106: 384-391. http:// dx.doi.org/10.1111/j.1471-4159.2008.05408.x

Honys D and Twell D (2003). Comparative analysis of the Arabidopsis pollen transcriptome. Plant Physiol. 132: 640-652. http://dx.doi.org/10.1104/pp.103.020925

Hsu SW, Liu MC, Zen KC and Wang CS (2014). Identification of the tapetum/microspore-specific promoter of the pathogenesis-related 10 gene and its regulation in the anther of Lilium longiflorum. Plant Sci. 215-216: 124-133. http://dx.doi.org/10.1016/j.plantsci.2013.11.006

Hyung D, Lee C, Kim JH, Yoo D, et al. (2014). Cross-family translational genomics of abiotic stress-responsive genes between Arabidopsis and Medicago truncatula. PLoS One 9: e91721. http://dx.doi.org/10.1371/journal.pone.0091721

Ji RQ, Song Q, Xin XF, Zhou X, et al. (2011). Isolation of fertility-related genes of multiple-allele-inherited male sterility in Brassica rapa ssp pekinensis by cDNA-AFLP. Genet. Mol. Res. 10: 4073-4083. http://dx.doi.org/10.4238/2011. December.5.8

Kaul ML (1988). Male sterility in higher plants. Springer-Verlag Press, Berlin.

Kim SS, Grienenberger E, Lallemand B, Colpitts CC, et al. (2010). LAP6/POLYKETIDE SYNTHASE A and LAP5/ POLYKETIDE SYNTHASE B encode hydroxyalkyl $\alpha$-pyrone synthases required for pollen development and sporopollenin biosynthesis in Arabidopsis thaliana. Plant Cell 22: 4045-4066. http://dx.doi.org/10.1105/ tpc. 110.080028

Kumagai T, Ito S, Nakamichi N, Niwa Y, et al. (2008). The common function of a novel subfamily of B-Box zinc finger proteins with reference to circadian-associated events in Arabidopsis thaliana. Biosci. Biotechnol. Biochem. 72: 1539-1549. http://dx.doi.org/10.1271/bbb.80041

Li L, Li Y, Song S, Deng H, et al. (2015). An anther development F-box (ADF) protein regulated by tapetum degeneration retardation (TDR) controls rice anther development. Planta 241: 157-166. http://dx.doi.org/10.1007/s00425-014$\underline{2160-9}$

Liu J, Pang C, Wei H, Song M, et al. (2014). Proteomic analysis of anthers from wild-type and photosensitive genetic male sterile mutant cotton (Gossypium hirsutum L.). BMC Plant Biol. 14: 390. http://dx.doi.org/10.1186/s12870$\underline{014-0390-4}$

Liu X, Huang J, Parameswaran S, Ito T, et al. (2009). The SPOROCYTELESS/NOZZLE gene is involved in controlling stamen identity in Arabidopsis. Plant Physiol. 151: 1401-1411. http://dx.doi.org/10.1104/pp.109.145896

Ma LC, Wang YR, Liu WX and Liu ZP (2013). Expression analysis of seed-specific genes in four angiosperm species with an emphasis on the unconserved expression patterns of homologous genes. Seed Sci. Res. 23: 223-231. http://dx.doi. org $/ 10.1017 / \mathrm{S} 0960258513000305$

Ma Y, Kang J, Wu J, Zhu Y, et al. (2015). Identification of tapetum-specific genes by comparing global gene expression of four different male sterile lines in Brassica oleracea. Plant Mol. Biol. 87: 541-554. http://dx.doi.org/10.1007/ $\underline{\mathrm{s} 11103-015-0287-0}$

Mahajan NS, Dewangan V, Lomate PR, Joshi RS, et al. (2015). Structural features of diverse Pin-II proteinase inhibitor genes from Capsicum annuum. Planta 241: 319-331. http://dx.doi.org/10.1007/s00425-014-2177-0

Manimaran P, Raghurami Reddy M, Bhaskar Rao T, Mangrauthia SK, et al. (2015). Identification of cis-elements and evaluation of upstream regulatory region of a rice anther-specific gene, OSIPP3, conferring pollen-specific expression in Oryza sativa (L.) ssp. indica. Plant Reprod. 28: 133-142. http://dx.doi.org/10.1007/s00497-015-0264-4

Matsuo Y, Arimura SI and Tsutsumi N (2013). Distribution of cellulosic wall in the anthers of Arabidopsis during microsporogenesis. Plant Cell Rep. 32: 1743-1750.

Pant BD, Burgos A, Pant P, Cuadros-Inostroza A, et al. (2015). The transcription factor PHR1 regulates lipid remodeling and triacylglycerol accumulation in Arabidopsis thaliana during phosphorus starvation. J. Exp. Bot. 66: 1907-1918. http://dx.doi.org/10.1093/jxb/eru535

Paul W, Hodge R, Smartt S, Draper J, et al. (1992). The isolation and characterisation of the tapetum-specific Arabidopsis thaliana A9 gene. Plant Mol. Biol. 19: 611-622. http://dx.doi.org/10.1007/s12298-012-0120-z

Pauls S, Goode DK, Petrone L, Oliveri P, et al. (2015). Evolution of lineage-specific functions in ancient cis-regulatory modules. Open Biol. 5: 150079. http://dx.doi.org/10.1098/rsob.150079

Qu Q, Xing YP, Liu WX, Xu XP, et al. (2008). Expression pattern and activity of six glutelin gene promoters in transgenic rice. J. Exp. Bot. 59: 2417-2424. http://dx.doi.org/10.1093/jxb/ern110

Raman S and Suguna K (2015). Functional characterization of heat-shock protein 90 from Oryza sativa and crystal structure of its N-terminal domain. Acta Crystallogr. F Struct. Biol. Commun. 71: 688-696. http://dx.doi.org/10.1107/ $\underline{\text { S2053230X15006639 }}$ 
Tian A, Cao J, Huang L, Yu X, et al. (2009). Characterization of a male sterile related gene BcMF15 from Brassica campestris ssp. chinensis. Mol. Biol. Rep. 36: 307-314. http://dx.doi.org/10.1007/s11033-007-9180-5

Town CD, Cheung F, Maiti R, Crabtree J, et al. (2006). Comparative genomics of Brassica oleracea and Arabidopsis thaliana reveal gene loss, fragmentation, and dispersal after polyploidy. Plant Cell 18: 1348-1359. http://dx.doi. org/10.1105/tpc. 106.041665

van Opijnen T, Kamoschinski J, Jeeninga RE and Berkhout B (2004). The human immunodeficiency virus type 1 promoter contains a CATA box instead of a TATA box for optimal transcription and replication. J. Virol. 78: 6883-6890. http:// dx.doi.org/10.1128/JVI.78.13.6883-6890.2004

Wang H, Lu Y, Jiang T, Berg H, et al. (2013). The Arabidopsis U-box/ARM repeat E3 ligase AtPUB4 influences growth and degeneration of tapetal cells, and its mutation leads to conditional male sterility. Plant J. 74: 511-523. http:// dx.doi.org/10.1111/tpj.12146

Wei LQ, Xu WY, Deng ZY, Su Z, et al. (2010). Genome-scale analysis and comparison of gene expression profiles in developing and germinated pollen in Oryza sativa. BMC Genomics 11: 338. http://dx.doi.org/10.1186/1471-2164$\underline{11-338}$

Wikström N, Savolainen V and Chase MW (2001). Evolution of the angiosperms: calibrating the family tree. Proc. Biol. Sci. 268: 2211-2220. http://dx.doi.org/10.1098/rspb.2001.1782

Wilson ZA and Zhang DB (2009). From Arabidopsis to rice: pathways in pollen development. J. Exp. Bot. 60: 1479-1492. http://dx.doi.org/10.1093/jxb/erp095

Wu Y, Min L, Wu Z, Yang L, et al. (2015). Defective pollen wall contributes to male sterility in the male sterile line 1355A of cotton. Sci. Rep. 5: 9608. http://dx.doi.org/10.1038/srep09608

Ye Q, Rosenberg SC, Moeller A, Speir JA, et al. (2015). TRIP13 is a protein-remodeling AAA+ ATPase that catalyzes MAD2 conformation switching. eLife 4: e07367.

Zhang XL and Zhang LG (2014). Molecular cloning and expression of the male sterility-related CtYABBY1 gene in flowering Chinese cabbage (Brassica campestris L. ssp chinensis var. parachinensis). Genet. Mol. Res. 13: 43364347. http://dx.doi.org/10.4238/2014.June. 10.1

Zhang ZL, Xie Z, Zou X, Casaretto J, et al. (2004). A rice WRKY gene encodes a transcriptional repressor of the gibberellin signaling pathway in aleurone cells. Plant Physiol. 134: 1500-1513. http://dx.doi.org/10.1104/pp.103.034967

Zhang ZS, Lu YG, Liu XD, Feng JH, et al. (2006). Cytological mechanism of pollen abortion resulting from allelic interaction of F1 pollen sterility locus in rice (Oryza sativa L.). Genetica 127: 295-302. http://dx.doi.org/10.1007/ $\underline{\text { s10709-005-4848-Z }}$

Zheng XM, Guo N, Gao TS and Zhang T (2015). Cloning and expression analysis of defensin genes from Brassica napus. Acta Agron. Sin. 41: 725-732. http://dx.doi.org/10.3724/SP.J.1006.2015.00725

Zhu H, Qian W, Lu X, Li D, et al. (2005). Expression patterns of purple acid phosphatase genes in Arabidopsis organs and functional analysis of AtPAP23 predominantly transcribed in flower. Plant Mol. Biol. 59: 581-594. http://dx.doi. org/10.1007/s11103-005-0183-0 